\title{
Estrogen modulates transactivations of SXR-mediated liver X receptor response element and CAR-mediated phenobarbital response element in HepG2 cells
}

\author{
Gyesik Min ${ }^{1}$ \\ Department of Pharmaceutical Engineering \\ Jinju National University \\ Jinju 660-758, Korea \\ ${ }^{1}$ Correspondence: Tel, 82-55-751-3396; \\ Fax, 82-55-751-3399; E-mail, g-min@jinju.ac.kr \\ DOI 10.3858/emm.2010.42.11.074
}

Accepted 14 September 2010

Available Online 27 September 2010

Abbreviations: CAR, constitutive androstane receptor; $C Y P$, cytochrome P450 gene; E2, 17- $\beta$ estradiol; ER, estrogen receptor; ERE, estrogen response element; GRIP, glucocorticoid receptor interacting protein; LRH, liver receptor homolog; LXR, liver $X$ receptor; LXREs, LXR response elements; MoxE2, moxestrol; PB, phenobarbital; PBRU, phenobarbital-responsive enhancer; PPAR, peroxisome proliferator activated receptor; $R X R$, retinoid $X$ receptor; SRC, steroid hormone receptor coactivator; SXR, steroid and xenobiotic receptor; TCPOBOP, 1,4-bis-(2-(3,5-dichloropyridoxyl)) benzene

\begin{abstract}
The nuclear receptors, steroid and xenobiotic receptor (SXR) and constitutive androstane receptor (CAR) play important functions in mediating lipid and drug metabolism in the liver. The present study demonstrates modulatory actions of estrogen in transactivations of SXR-mediated liver $X$ receptor response element (LXRE) and CAR-mediated phenobarbital response element (PBRU). When human estrogen receptor (hER $\alpha$ ) and SXR were exogenously expressed, treatment with either rifampicin or corticosterone promoted significantly the SXR-mediated transactivation of LXRE reporter gene in HepG2. However, combined treatment with estrogen plus either rifampicin or corticosterone resulted in less than $\mathbf{5 0} \%$ of the mean values of the transactivation by rifampicin or corticosterone alone. Thus, it is suggested that estrogen may repress the SXR-mediated transactivation of LXRE via functional cross-talk between ER and SXR. The CAR-mediated transactivation of PBRU was stimulated by $h E R \alpha$ in the absence of estrogen. However, the potentiation by CAR agonist, TCPOBOP, was significantly repressed
\end{abstract}

by moxestrol in the presence of ER. Thus, ER may play both stimulatory and inhibitory roles in modulating CAR-mediated transactivation of PBRU depending on the presence of their ligands. In summary, this study demonstrates that estrogen modulates transcriptional activity of SXR and CAR in mediating transactivation of LXRE and PBRU, respectively, of the nuclear receptor target genes through functional cross-talk between ER and the corresponding nuclear receptors.

Keywords: constitutive androstane receptor; estrogen; liver $\mathrm{X}$ receptor; phenobarbital; pregnane $\mathrm{X}$ receptor; transcriptional activation

\section{Introduction}

Estrogen plays important biological functions not only in the development of female reproduction and cellular proliferation but also in lipid metabolism and biological homeostasis in different tissues of body (Archer et al., 1986; Croston et al., 1997; Blum and Cannon, 2001; Deroo and Korach, 2006; Glass, 2006). Estrogen action is mediated by the nuclear estrogen receptor (ER), which is a ligand-dependent transcription factor and consists of different regulatory domains with distinct biological functions (Evans, 1988; Beato, 1989). Ligand-bound ER either binds to estrogen response element (ERE) directly or interacts indirectly to the DNA through protein-protein interactions with other transcriptional factors in estrogen-responsive target genes (Paech et al., 1997). Ligand binding induces a conformational change of the ER and recruits different sets of coactivators or corepressors that determine biological activity by changing the transcriptional responses according to the physiological needs (Katzenellenbogen and Katzenellenbogen, 2000).

Recent studies (Min et al., 2002b) demonstrated that the action of ER in transcriptional activity can be modulated by functional cross-talk between ER and other nuclear receptors. Specifically, the xenobiotic nuclear receptors, constitutive androstane receptor (CAR) and steroid and xenobiotic receptor (SXR), inhibit ER-mediated transactivation. These xenobiotic receptors play important roles in modulating steroid hormone homeostasis and drug 
metabolism by mediating cellular physiological responses not only to the endogenous compounds such as hormones but also to the exogenous chemical compounds such as drugs and environmental pollutants.

The orphan nuclear receptor CAR mediates induction of cytochrome P450 (CYP)2B genes, which are steroid hydroxylases, by the classical inducer of drug metabolism, phenobarbital (PB), in the liver (Wei et al., 2000; Min et al., 2002a, $2002 b)$. CAR is sequestered in the cytoplasm and upon exposure to agonists, such as $\mathrm{PB}$ and 1,4-bis-(2-(3,5-dichloropyridoxyl))benzene (TCPOBOP) (Tzameli et al., 2000), translocated into the nucleus (Kawamoto et al., 1999; Min et al., 2002a), where it binds to its cognate recognition sites called nuclear receptor (NR)-1 and NR-2 as a CAR/retinoid $X$ receptor ( $R X R)$ heterodimer in the phenobarbital-responsive enhancer (PBRU) of the CYP2B genes (Trottier et al., 1995; Honkakoski et al., 1998; Kawamoto et al., 1999; Kim et al., 2001; Min et al., 2002a). Although transcriptional activity of CAR is ligand-independent, some of CAR inducers such as TCPOBOP can bind CAR directly and enhance its transcriptional activity by promoting the interaction of CAR with the coactivators, steroid hormone receptor coactivator (SRC)-1 (Forman et al., 1998; Moore et al., 2000; Tzameli et al., 2000) and glucocorticoid receptor interacting protein (GRIP)-1 (Min et al., 2002a).

The steroid and xenobiotic nuclear receptor SXR can modulate transcription in response to diverse natural and synthetic compounds with a broad spectrum of ligand specificity and low- affinity. It is highly expressed in liver, the major expression site of steroid and xenobiotic-metabolizing enzymes, and intestine known to play important role in the first stage of metabolism of dietary compounds (Kolars et al., 1991; Blumberg et al., 1998). SXR forms a heterodimer with RXR that can bind to and induce transcription from the response elements of cytochrome P450 genes including CYP3A4 and CYP2A that are involved in steroid and xenobiotic metabolism (Gonzalez, 1992; Blumberg et al., 1998). SXR is activated by a variety of xenobiotic compounds, including drugs such as rifampicin, steroid receptor agonists and antagonists such as estrogen and tamoxifen respectively, and bioactive dietary compounds such as phytoestrogens (Blumberg et al., 1998). Thus, it was hypothesized that SXR acting as a broad sensor nuclear receptor may monitor aggregate levels of diverse inducers in order to modulate expression of xenobiotic-metabolizing enzymes (Blumberg et al., 1998). The structural diversity of compounds that activate $S X R$ and the fact that some of these compounds can also interact with classic steroid hormone receptors suggest that multiple signal cross-talk mechanisms might be involved.

The liver $X$ receptor ( $L X R)$ is an orphan nuclear receptor that plays important physiological roles in the regulation of lipid metabolism. LXR serves as a sensor of cellular cholesterol and regulate the expression of genes such as CYP7A1 involved in the maintenance of cholesterol homeostasis and fatty acid biosynthesis (Glass, 2006). LXR recognizes DR-4 LXR response elements (LXREs) as a heterodimer with $R X R$, and 9-cis retinoic acid was reported to be a potent activator of LXR transcriptional activity (Willy et al., 1995; Glass, 2006: Matsukuma et al., 2007). A previous report also demonstrated that LXR heterodimerizes with peroxisome proliferator activated receptor (PPAR) to regulate gene expression suggesting possible interactions with other related nuclear receptors (Miyata et al., 1996).

Nuclear receptors can positively or negatively regulate gene expression by several mechanisms. Many nuclear receptors can inhibit signal-dependent activation of promoters by other classes of transcription factors in a ligand-dependent manner. An example of this is the ability of the glucocorticoid receptor to inhibit activation of inflammatory response genes by signal-dependent transcription factors such as activator protein-1 and NF-кB (De Bosscher et al., 2003; Glass, 2006). The nuclear receptors are tethered to target promoters through protein-protein interactions (Glass, 2006). This trans-repression accounts for a majority of the anti-inflammatory actions of synthetic glucocorticoids such as dexamethasone (Glass, 2006) and may be an important mechanism by which other nuclear receptor ligands such as estrogens exert modulatory effects.

The liver is the major organ that metabolizes steroids and xenobiotic chemical compounds, and one of the target organs for estrogen action in the body (Min et al., 2002b). ER and the orphan nuclear receptors, SXR and CAR, and their heterodimeric partner $\mathrm{RXR}$ are all expressed in the liver (Kliewer et al., 1999). Although several studies have been reported on the modulation of ER transcriptional activity by other nuclear receptors (Glass et al., 1988; Kraus et al., 1995; Nuñez et al., 1997; Duan et al., 1999; Ricci et al., 1999; Min et al., 2002b), it is not known whether ER can modulate transcriptional activities of the xenobiotic nuclear receptors, SXR or CAR. Accordingly, this study examined modulatory actions of estrogen in transactivations of both SXRmediated LXRE and CAR-mediated PBRU in a human hepatoma cell line, HepG2. 


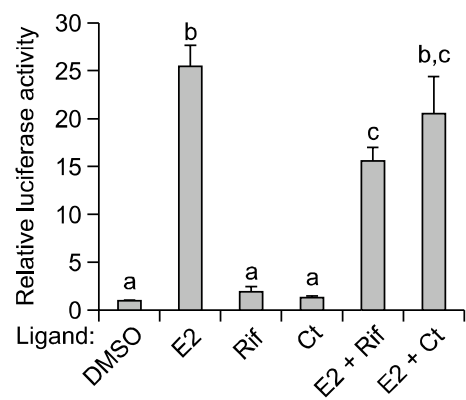

Figure 1. Effects of estrogen, rifampicin, and corticosterone on the synthetic (4ERE)-tk-luciferase transactivation in HepG2 cells. HepG2 cells were transfected with $250 \mathrm{ng}$ of 4ERE-tk-luciferase reporter vector and $10 \mathrm{ng}$ of pRLSV40 as an internal control for transfection efficiency in the presence of pCMX-RXR ( $1 \mathrm{ng})$, pCMV-hER $\alpha(5 \mathrm{ng})$, and pCMX-SXR ( 5 $\mathrm{ng}$ ). The ligands were added for $24 \mathrm{~h}$ after transfection as indicated: 10 $\mathrm{nM}$ of $17 \beta$-estradiol (E2) for ER and $10 \mu \mathrm{M}$ of rifampicin (Rif) or $10 \mu \mathrm{M}$ of corticosterone (Ct) for SXR. The cells were harvested for dual luciferase assays. The values for firefly luciferase were normalized by dividing by Renilla luciferase values. The standard errors of the mean were calculated from four independent transfection experiments. Bars with different superscript letters differ significantly $(P<0.05)$.

\section{Results}

\section{Estrogen induces ER-mediated transactivation of a synthetic ERE-driven promoter in HepG2}

In order to verify that estrogen can induce ERmediated transactivation of a cognate synthetic ERE in the cell culture system, transient transfection experiments were carried out in a human hepatoma cell line. HepG2 cells were cotransfected with expression plasmids of a human ER and the orphan nuclear receptors, SXR and RXR, and a luciferase reporter plasmid containing $a$ synthetic four ERE (4ERE) in the upstream of the thymidine kinase promoter. As shown in Figure 1, treatment with $10 \mathrm{nM} 17-\beta$ estradiol (E2) markedly stimulated (25-fold) the ER-mediated expression of the luciferase reporter gene. In contrast, treatment with either $10 \mu \mathrm{M}$ rifampicin or $10 \mu \mathrm{M}$ corticosterone, the ligands for SXR, did not transactivate the (4ERE)-tk-luciferase reporter (Figure 1). In addition, consistent with a previous report (Min et al., 2002b), co-treatment with rifampicin inhibited the estrogen stimulated ER transactivation by $40 \%$ (Figure 1). However, activation of SXR by corticosterone did not affect the estrogen stimulated ER transcriptional activity. These results indicate that estrogen induces ER-mediated transactivation of ERE in HepG2 cells and thus can be used as a functional control to examine the effects of ER on transcriptional modulations of the xenobiotic nuclear receptors, such as SXR and CAR, in context to their target response elements in this assay system.

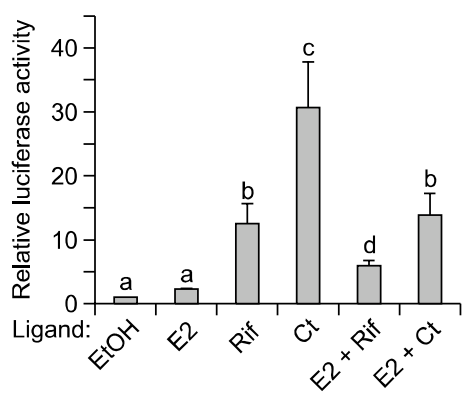

Figure 2. Effects of estrogen, rifampicin, and corticosterone on the LXRE transactivation in HepG2 cells. HepG2 cells were transfected with $250 \mathrm{ng}$ of (LXRE) $)_{3}$-tk-luciferase vector and $10 \mathrm{ng}$ of pRLSV40 as an internal control for transfection efficiency in the presence of pCMX-SXR $(10 \mathrm{ng})$ and $\mathrm{pCMV}$-hER $\alpha(2.5 \mathrm{ng})$. The ligands were added for $24 \mathrm{~h}$ after transfection as indicated: $10 \mathrm{nM}$ of $17 \beta$-estradiol (E2) for ER and $10 \mu \mathrm{M}$ of rifampicin (Rif) or $10 \mu \mathrm{M}$ of corticosterone (Ct) for SXR. The cells were harvested for dual luciferase assays. The values for firefly luciferase were normalized by dividing by Renilla luciferase values. The standard errors of the mean were calculated from six independent transfection experiments. Bars with different superscript letters differ significantly $(P$ $<0.05)$.

\section{Activated ER inhibits transactivation of LXRE by ligand-activated SXR in HepG2}

This study also examined whether SXR can transactivate LXRE in the transient transfection system of cultured HepG2. Whereas ER did not transactivate a synthetic (LXRE) $)_{3}$-tk-luciferase reporter gene, the ligand-activated SXR either by rifampicin or corticosterone significantly induced transcriptional activation of the LXRE-driven promoter by 12- and 30 -fold respectively (Figure 2 ). This study then determined if estrogen can modulate the SXR-mediated transactivation of LXRE via functional cross-talk between ER and the ligand-activated SXR. Treatment with E2 caused a significant reduction in the SXR-mediated transactivation of LXRE by rifampicin or corticosterone (Figure 2). Co-treatments with estrogen resulted in less than $50 \%$ in mean values of the transactivation by rifampicin and corticosterone alone.

\section{PBRU is transactivated by CAR but not by ER}

In order to examine the effects of estrogen on CAR-mediated transactivation of PBRU, this study first determined if estrogen can induce PBRU transactivation in the absence of CAR transfected in transient transfection system. When HepG2 cells were cotransfected with hER and a reporter plasmid containing a synthetic four repeats of NR1 site of CYP2B1 PBRU (CYP2B1NR1 ${ }_{4}$ ) in the upstream of the CYP2C1 promoter, treatment with $10 \mathrm{nM}$ moxestrol (MoxE2) did not induce transactivation of the synthetic PBRU NR1 suggesting that PBRU 


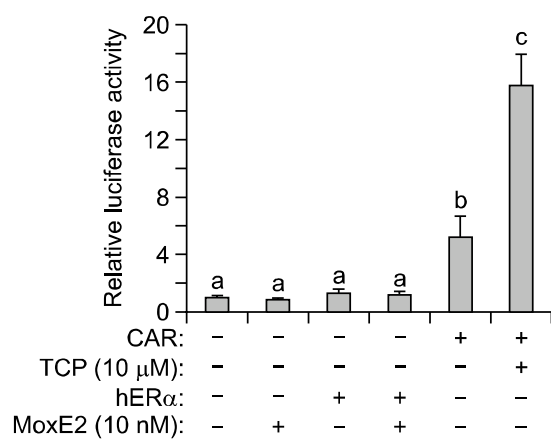

Figure 3. Transactivation of CYP2B1(NR-1) 4 is mediated by CAR. HepG2 cells were transfected with $2 \mu \mathrm{g}$ of CYP2B1(NR-1) 4 -2C1-luciferase reporter and $10 \mathrm{ng}$ of pRLSV40 in the absence and presence of CMV-hER $\alpha(10 \mathrm{ng})$ and/or pcDNA3-CAR (1 $\mathrm{ng})$. The ligands were added for $24 \mathrm{~h}$ after transfection as indicated: $10 \mu \mathrm{M}$ of TCPOBOP (TCP) for CAR and $10 \mathrm{nM}$ of MoxE2 for ER. The cells were harvested for dual luciferase assays. The values for firefly luciferase were normalized by dividing by Renilla luciferase values. The standard errors of the mean were calculated from six independent transfection experiments. Bars with different superscript letters differ significantly $(P<0.05)$.

is not transactivated by ER in HepG2 cells (Figure 3). In contrast, as reported previously, transfection of CAR induced PBRU transactivation and treatment with $10 \mu \mathrm{M}$ TCPOBOP further enhanced the CAR-mediated PBRU transactivation by 3-fold (Figure 3). These results indicate that transactivation of PBRU can be used to examine the possible cross-talk between CAR and ER.

\section{ER can modulate CAR-mediated transactivation of PBRU in HepG2}

This study further determined if ER can play a regulatory role in CAR-mediated transactivation of PBRU. SThe CAR-mediated transactivation of CYP2B1PBRU-2C1-luciferase was not affected by MoxE2 in the absence of hER $\alpha$. Whereas, transfection with $h E R \alpha$ without estrogen treatment stimulated the CAR-mediated reporter activity in the absence of the CAR agonist, TCPOBOP (Figure 4). Treatment with the estrogen, however, abolished the stimulatory effect of $h E R \alpha$ on the CAR-mediated transactivation. More interestingly, the potentiation of the CAR-mediated transactivation of PBRU by TCPOBOP was significantly repressed by MoxE2 only in the presence of hER $\alpha$ (Figure 4).

\section{Discussion}

Recent studies (Deroo and Korach, 2006; Glass, 2006) indicate that multiple molecular mechanisms may be used by different members of the nuclear

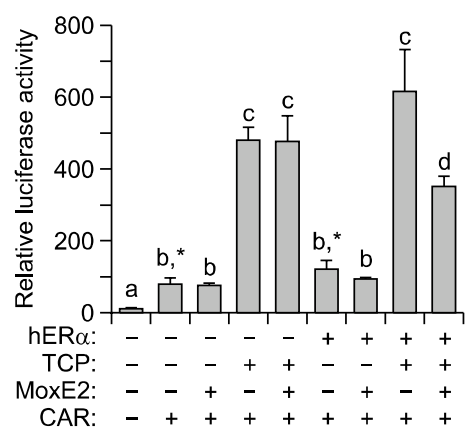

Figure 4. Estrogen receptor may play both stimulatory and inhibitory roles in CAR-mediated transactivation of PBRU depending on the presence of estrogen and CAR agonist. HepG2 cells were transfected with 2 $\mu \mathrm{g}$ of CYP2B1PBRU-2C1-luciferase reporter and $10 \mathrm{ng}$ of pRLSV40 in the absence and presence of CMV-hER $\alpha(10 \mathrm{ng})$ and/or pcDNA3-CAR $(1 \mathrm{ng})$. The ligands were added for $24 \mathrm{~h}$ after transfection as indicated: $10 \mu \mathrm{M}$ of TCPOBOP (TCP) for CAR and $10 \mathrm{nM}$ of MoxE2 for ER. The cells were harvested for dual luciferase assays. The values for firefly luciferase were normalized by dividing by Renilla luciferase values. The standard errors of the mean were calculated from six independent transfection experiments. Bars with different superscript letters differ significantly $(P<0.05)$. *, Indicates a difference $(P<0.05)$ from each other.

receptor family to regulate signal-dependent gene activation. This study demonstrates that ER may modulate transcriptional activity of xenobiotic nuclear receptors, SXR and CAR.

Both rifampicin and corticosterone stimulated the SXR-mediated transactivation of LXRE and estrogen had repressive effects on the ligandbound SXR transcriptional activity. These results are consistent with a previous report that corticosterone is a potent ligand specific for SXR and not for ER (Blumberg et al., 1998), and suggest a functional cross-talk between ER and SXR in HepG2 cells. This regulatory role of estrogen in LXRE transactivation may have physiological implications in the bile acid homeostasis in the liver. Estrogens have been reported to cause hepatotoxicity such as intrahepatic cholestasis during pregnancy (Schreiber and Simon, 1983). A recent study demonstrated that $E R \alpha$ mediates alterations of bile acid biosynthesis and repression of multiple hepatic bile acid and cholesterol transporters that contribute to liver damage (Yamamoto et al., 2006). Bile acid homeostasis is tightly regulated by multiple nuclear receptors including SXR and CAR in physiological and pathological conditions (Karpen, 2002; Francis et al., 2003; Guo et al., 2003). The activation of SXR inhibits production of bile acids by inhibiting expression of CYP7A1, the ratelimiting enzyme for bile acid biosynthesis, and inducing organic anion transporting polypeptide 2 which increases bile acid uptake from sinusoidal blood to the hepatocytes (Staudinger et al., 2001; 
Xie et al., 2001). CAR induces sulfotransferase $2 A 1$, which is an enzyme to sulfate hydroxy-bile acids and steroids, and multidrug resistance-associated protein 4 in order to prevent toxic bile acid accumulation (Assem et al., 2004). Double nullmouse lacking SXR and CAR was reported to have a more severe disruption of bile acids and cholesterol homeostasis suggesting preventive roles of SXR and CAR in the cholestatic condition (Stedman et al., 2005; Uppal et al., 2005). In addition, an $\mathrm{ER} \alpha$ antagonist, tamoxifen, decreased alkaline phosphatase levels in primary biliary cirrhosis patients suggesting that inhibition of ER $\alpha$ action may have anti-cholestasis effect (Invernizzi et al., 2004; Reddy et al., 2004). Thus, regulation of genes involved in the bile acid homeostasis by ER $\alpha$ plays an important role in estrogen-induced hepatotoxicity. The molecular mechanisms whereby $\mathrm{ER} \alpha$ regulates hepatic genes in bile acid homeostasis are not well understood. This study may provide an insight into a possible molecular mechanism whereby estrogen regulate expression of these genes. Estrogen may exert its effects through functional crosstalk between ER and other orphan nuclear receptors such as SXR and LXR which transactivate LXRE present in their target genes. This is also supported by a previous report that some of the genes involved in bile acid metabolism were regulated by LXR and liver receptor homolog (LRH)-1 (Francis et al., 2003; Yamamoto et al., 2006). However, whether ER modulation of the SXR-mediated transactivation of LXRE involves either direct or indirect interactions between these nuclear receptors remains to be elucidated.

This study also demonstrates that ER may play both inhibitory and stimulatory roles in CAR-mediated transactivation of PBRU depending on the presence of estrogen and CAR ligand. The finding that the estrogen-bound ER $\alpha$ repressed the CARmediated PBRU transactivation enhanced by the CAR agonist may suggest estrogen's regulatory role in the xenobiotic nuclear receptor function by cross-talk between activated ER and CAR. This cross-talk mechanism of the nuclear receptor regulation is consistent with a previous report that ER-mediated transactivation of estrogen responsive genes is repressed by inhibitory cross-talk between ER and CAR (Min et al., 2002b). The molecular mechanisms whereby estrogen modulates transcriptional activity of CAR are not currently understood. However, two lines of evidence led to speculate that ER may repress the CARmediated transcriptional activity by squelching coactivators in the hepatocytes. Both the orphan nuclear receptor and ER were reported to have common components in their regulatory pathways (Moore et al., 2000; Tzameli et al., 2000; Min et al., 2002a). For example, both CAR and ER interact with the p160 coactivators, SRC-1 and GRIP-1, as the important transcriptional coactivators. Furthermore, a previous study (Min et al., 2002b) provided evidence that CAR antagonizes the ER-mediated transcriptional activity by competitively inhibiting the interaction of ER with the p160 coactivators which are essential for ER action.

There may also be other potential mechanisms by which ER represses CAR-mediated transcriptional activity. Ligand-bound ER may interact directly with CAR and form an inactive complex which in turn blocks the dimerization interface with RXR and/or coactivator interacting domain and thus inhibition of CAR binding to PBRU. However, this possibility may be less likely to explain the cross-regulation since the previous studies (Min et al., 2002b) showed that CAR did not interact with ER bound to ERE. On the other hand, ER may also compete with CAR for binding to the PBRU. However, the MoxE2-bound ERa did not induce transactivation of the synthetic PBRU NR1 in transient transfection system of HepG2 (Figure 4) suggesting that ER does not bind to PBRU. Thus, the transcriptional activity of one nuclear receptor may not only be regulated by its cognate ligand but also be modulated by other nuclear receptors through functional cross-talks between the receptors.

It is interesting to find that the CAR-mediated PBRU transactivation was also stimulated by the exogenous hER in the absence of estrogen. It may be that the different conformational structure of the ER from that of estrogen-bound state may recruit and/or release different sets of coregulators to bring about its regulatory effects on transcriptional activity of CAR. However, the mechanism of ER action in the absence of ligand in modulating transcriptional activity of other nuclear receptors such as CAR remains to be elucidated.

In summary, this study demonstrates that estrogen modulates transcriptional activity of SXR and CAR in mediating transactivation of LXRE and PBRU, respectively, of the nuclear receptor target genes through functional cross-talk between ER and the corresponding nuclear receptors.

\section{Methods}

\section{Ligand and plasmid}

The ligands for ER, E2 and MoxE2, and for SXR, rifampicin and corticosterone were purchased from Sigma Chemical Company (St. Louis, IL). Reporter constructs of CYP2B1PBRU-2C1-luciferase, CYP2B1(NR-1) 4 -2C1-luciferase, and 4ERE-tk-luciferase that were described before (Liu et al., 1998; Kim et al., 2001; Min et al., 2002a, 2002b) 
and (LXRE) $)_{3}$-tk-luciferase were obtained from J. K. Kemper. The ligand for CAR, TCPOBOP, was obtained from $B$. Kemper. Mouse CAR1 cDNA was isolated from a mouse liver CDNA library by polymerase chain reaction and verified by sequencing. For expression of CAR in mammalian cells, a BamH1/EcoR1 fragment containing the CAR1 cDNA isolated from pGEX2TK-CAR was inserted into pcDNA3 (Invitrogen) digested with the same enzymes to produce pcDNA3-CAR. The expression plasmids for $h E R \alpha, R X R$, and SXR were obtained from J. K. Kemper.

\section{Cell culture}

Human HepG2 cells (American Type Culture Collection [ATCC] HB8065) were maintained in $5 \% \mathrm{CO}_{2}$ at $37^{\circ} \mathrm{C}$ in phenol-red free Dulbecco's modified Eagle's medium (DMEM; Gibco, Grand Island, NY) supplemented with 10\% charcoal dextran-striped calf serum, 100 units $/ \mathrm{ml}$ penicillin, and $100 \mu \mathrm{g} / \mathrm{ml}$ streptomycin.

\section{Transfection}

For transfection, the human hepatoma cells were cultured until about $90 \%$ confluence and were transfected using LipofectAMINE 2000 (Invitrogen). Cells were seeded in 24-well plates, and $250 \mathrm{ng}$ of 4ERE-tk-luciferase, $250 \mathrm{ng}$ of $(\mathrm{LXRE})_{3}$-tk-luciferase, $2 \mu \mathrm{g}$ of either CYP2B1(NR-1) ${ }_{4}-2 \mathrm{C} 1$ luciferase or CYP2B1PBRU-2C1-luciferase, $10 \mathrm{ng}$ of $\mathrm{pRL}$ SV40 for measuring the transfection efficiency, and varying amounts of nuclear receptor expression plasmids for $\mathrm{hER} \alpha$ (2.5-25 ng), RXR (1 ng), CAR (1 ng), and SXR (5-50 ng) were added to each well. In order to examine the effects of ligand for each receptor, $10 \mathrm{nM}$ of E2, $10 \mathrm{nM}$ of MoxE2, 10 $\mu \mathrm{M}$ of TCPOBOP, and $10 \mu \mathrm{M}$ of rifampicin and corticosterone were added. The cells were incubated for 16-24 $\mathrm{h}$ after transfection, fresh medium containing the ligands was added, and the cells were incubated an additional $24 \mathrm{~h}$.

\section{Dual luciferase assay}

The cells were lysed and luciferase activities were determined by the dual luciferase reporter assay system (Promega Biotech). For each sample, the background of extracts from untreated cells was subtracted and the firefly luciferase values were normalized by dividing by the Renilla luciferase values.

\section{Statistical analysis}

Data are expressed as means \pm SE. Statistical significance was determined by Student's $t$-test. A value of $P<$ 0.05 was considered statistically significant.

\section{Acknowledgments}

The author thanks Drs. B. Kemper and J. K. Kemper for providing plasmids and ligand for this study.

\section{References}

Archer TK, Tam SP, Deeley RG. Kinetics of estrogendependent modulation of apolipoprotein A-I synthesis in human hepatoma cells. J Biol Chem 1986;261:5067-74

Assem M, Schuetz EG, Leggas M, Sun D, Yasuda K, Reid G, Zelcer N, Adachi M, Strom S, Evans RM, Moore DD, Borst P, Schuetz JD. Interactions between hepatic Mrp4 and Sult2a as revealed by the constitutive androstane receptor and Mrp4 knockout mice. J Biol Chem 2004;279:22250-7

Beato M. Gene regulation by steroid hormones. Cell 1989; 56:335-44

Blum A, Cannon RO 3rd. Selective ER modulator effects on serum lipoproteins and vascular function in postmenopausal women and in hypercholesterolemic men. Ann N Y Acad Sci 2001;949:168-74

Blumberg B, Sabbagh W Jr, Juguilon $\mathrm{H}$, Bolado J Jr, van Meter CM, Ong ES, Evans RM. SXR, a novel steroid and xenobiotic-sensing nuclear receptor. Genes Dev 1998;12: 3195-205

Croston GE, Milan LB, Marschke KB, Reichman M, Briggs MR. Androgen receptor-mediated antagonism of estrogen-dependent low density lipoprotein receptor transcription in cultured hepatocytes. Endocrinology 1997;138:3779-86

De Bosscher K, Vanden Berghe W, Haegeman G. The interplay between the glucocorticoid receptor and nuclear factor-kappaB or activator protein-1: molecular mechanisms for gene repression. Endocr Rev 2003;24:488-522

Deroo BJ, Korach KS. Estrogen receptors and human disease. J Clin Invest 2006;116:561-70

Duan R, Porter W, Samudio I, Vyhlidal C, Kladde M, Safe S. Transcriptional activation of c-fos protooncogene by 17beta-estradiol: mechanism of aryl hydrocarbon receptor-mediated inhibition. Mol Endocrinol 1999;13:1511-21

Evans R. The steroid and thyroid hormone receptor superfamily. Science 1988;240:889-95

Forman BM, Tzameli I, Choi HS, Chen J, Simha D, Seol W, Evans RM, Moore DD. Androstane metabolites bind to and deactivate the nuclear receptor CAR-beta. Nature 1998; 395:612-15

Francis GA, Fayard E, Picard F, Auwerx J. Nuclear receptors and the control of metabolism. Annu Rev Physiol 2003; 65:261-311

Glass CK, Holloway JM, Devary OV, Rosenfeld MG. The thyroid hormone receptor binds with opposite transcriptional effects to a common sequence motif in thyroid hormone and estrogen response elements. Cell 1988;54:313-23

Glass CK. Going nuclear in metabolic and cardiovascular disease. J Clin Invest 2006;116:556-60

Gonzalez FJ. Human cytochromes P450: problems and prospects. Trends Pharmacol Sci 1992;13:346-52

Guo GL, Lambert G, Negishi M, Ward JM, Brewer HB Jr, Kliewer SA, Gonzalez FJ, Sinal CJ. Complementary roles of farnesoid $X$ receptor, pregnane $X$ receptor, and constitutive 
androstane receptor in protection against bile acid toxicity. J Biol Chem 2003;278:45062-71

Honkakoski P, Zelko I, Sueyoshi T, Negishi M. The nuclear orphan receptor CAR-retinoid $X$ receptor heterodimer activates the phenobarbital-responsive enhancer module of the CYP2B gene. Mol Cell Biol 1998;18:5652-8

Invernizzi P, Alvaro D, Crosignani A, Gaudio E, Podda M. Tamoxifen in treatment of primary biliary cirrhosis. Hepatology 2004;39:1175-6

Karpen SJ. Nuclear receptor regulation of hepatic function. J Hepatol 2002;36:832-50

Katzenellenbogen BS, Katzenellenbogen JA. Estrogen receptor transcription and transactivation: Estrogen receptor alpha and estrogen receptor beta: regulation by selective estrogen receptor modulators and importance in breast cancer. Breast Cancer Res 2000;2:335-44

Kawamoto T, Sueyoshi T, Zelko I, Moore R, Washburn K, Negishi M. Phenobarbital-responsive nuclear translocation of the receptor CAR in induction of the CYP2B gene. Mol Cell Biol 1999;19:6318-22

Kim J, Min G, Kemper B. Chromatin assembly enhances binding to the CYP2B1 phenobarbital-responsive unit (PBRU) of nuclear factor-1, which binds simultaneously with constitutive androstane receptor (CAR)/retinoid $X$ receptor (RXR) and enhances CAR/RXR-mediated activation of the PBRU. J Biol Chem 2001;276:7559-67

Kliewer SA, Lehmann JM, Willson TM. Orphan nuclear receptors: shifting endocrinology into reverse. Science 1999;284:757-60

Kolars JC, Awni WM, Merion RM, Watkins PB. First-pass metabolism of cyclosporin by the gut. Lancet 1991;338: 1488-90

Kraus WL, Weis KE, Katzenellenbogen BS. Inhibitory cross-talk between steroid hormone receptors: differential targeting of estrogen receptor in the repression of its transcriptional activity by agonist-and antagonist-occupied progestin receptors. Mol Cell Biol 1995;15:1847-57

Liu S, Park Y, Rivera-Rivera I, Li H, Kemper B. Nuclear factor-1 motif and redundant regulatory elements comprise phenobarbital-responsive enhancer in CYP2B1/2. DNA Cell Biol 1998;17:461-70

Matsukuma KE, Wang L, Bennett MK, Osborne TF. A key role for orphan nuclear receptor liver receptor homologue-1 in activation of fatty acid synthase promoter by liver $\mathrm{X}$ receptor. J Biol Chem 2007;282:20164-71

Min G, Kemper JK, Kemper B. Glucocorticoid receptorinteracting protein 1 mediates ligand-independent nuclear translocation and activation of constitutive androstane receptor in vitro. J Biol Chem 2002a;277:26356-63

Min G, Kim H, Bae Y, Petz L, Kemper JK. Inhibitory cross-talk between estrogen receptor (ER) and constitutively activated androstane receptor (CAR). J Biol Chem 2002b;277: 34626-33

Miyata KS, McCaw SE, Patel HV, Rachubinski RA, Capone JP. The orphan nuclear hormone receptor LXR alpha interacts with the peroxisome proliferator-activated receptor and inhibits peroxisome proliferator signaling. J Biol Chem 1996;271:9189-92

Moore LB, Parks DJ, Jones SA, Bledsoe RK, Consler TG, Stimmel JB, Goodwin B, Liddle C, Blanchard SG, Willson TM, Collins JL, Kliewer SA. Orphan nuclear receptors constitutive androstane receptor and pregnane $X$ receptor share xenobiotic and steroid ligands. J Biol Chem 2000;275: 15122-7

Nuñez SB, Medin JA, Braissant O, Kemp L, Wahli W, Ozato $\mathrm{K}$, Segars JH. Retinoid X receptor and peroxisome proliferator-activated receptor activate an estrogen responsive gene independent of the estrogen receptor. Mol Cell Endocrinol 1997; 127:27-40

Paech K, Webb P, Kuiper GG, Nilsson S, Gustafsson J, Kushner PJ, Scanlan TS. Differential ligand activation of estrogen receptors ERalpha and ERbeta at AP1 sites. Science 1997;277:1508-10

Reddy A, Prince M, James OF, Jain S, Bassendine MF. Tamoxifen: a novel treatment for primary biliary cirrhosis? Liver Int 2004;24:194-7

Ricci MS, Toscano DG, Mattingly CJ, Toscano WA Jr. Estrogen receptor reduces CYP1A1 induction in cultured human endometrial cells. J Biol Chem 1999;274:3430-8

Schreiber AJ, Simon FR. Estrogen-induced cholestasis: clues to pathogenesis and treatment. Hepatology 1983; 3:607-13

Staudinger JL, Goodwin B, Jones SA, Hawkins-Brown D, MacKenzie KI, LaTour A, Liu Y, Klaassen CD, Brown KK, Reinhard J, Willson TM, Koller BH, Kliewer SA. The nuclear receptor $\mathrm{PXR}$ is a lithocholic acid sensor that protects against liver toxicity. Proc Natl Acad Sci USA 2001;98:3369-74

Stedman CA, Liddle C, Coulter SA, Sonoda J, Alvarez JG, Moore DD, Evans RM, Downes M. Nuclear receptors constitutive androstane receptor and pregnane $X$ receptor ameliorate cholestatic liver injury. Proc Natl Acad Sci USA 2005;102:2063-8

Trottier E, Belzil A, Stoltz C, Anderson A. Localization of a phenobarbital-responsive element (PBRE) in the 5'-flanking region of the rat CYP2B2 gene. Gene 1995;158:263-8

Tzameli I, Pissios P, Schuetz EG, Moore DD. The xenobiotic compound 1,4-bis[2-(3,5-dichloropyridyloxy)]benzene is an agonist ligand for the nuclear receptor CAR. Mol Cell Biol 2000;20:2951-8

Uppal H, Toma D, Saini SP, Ren S, Jones TJ, Xie W. Combined loss of orphan receptors PXR and CAR heightens sensitivity to toxic bile acids in mice. Hepatology 2005; 41:168-76

Wei P, Zhang J, Egan-Hafley M, Liang S, Moore DD. The nuclear receptor CAR mediates specific xenobiotic induction of drug metabolism. Nature 2000;407:920-3

Willy PJ, Umesono K, Ong ES, Evans RM, Heyman RA, Mangelsdorf DJ. LXR, a nuclear receptor that defines a distinct retinoid response pathway. Genes Dev 1995;9: 1033-45

Xie W, Radominska-Pandya A, Shi Y, Simon CM, Nelson MC, Ong ES, Waxman DJ, Evans RM. An essential role for 
nuclear receptors SXR/PXR in detoxification of cholestatic bile acids. Proc Natl Acad Sci USA 2001;98:3375-80

Yamamoto Y, Moore R, Hess HA, Guo GL, Gonzalez FJ,
Korach KS, Maronpot RR, Negishi M. Estrogen receptor alpha mediates 17alpha-ethynylestradiol causing hepatotoxicity. J Biol Chem 2006;281:16625-31 